\title{
Prolong Fever with Murmur in Heart in a patient with History of Intravenous Drug Abuse- A Case Report
}

\author{
KK Islam, N Kar, M Ullah \\ Department of Cardiology, NICVD, Dhaka.
}

Keywords:

Infective

endocarditis,

Tricuspid valve

IVDU

\begin{abstract}
:
Right sided endocarditis accounts for $10 \%$ of all IE in population based surveys ${ }^{1}$ and a higher proportion of IE in injection drug users. Among injection drug users presenting with fever, 13\% will have Echocardiographic evidence of IE. ${ }^{2}$ Staphylococcal endocarditis in injection drug users is now the dominant form of the disease in many urban communities where there is a high incidence of injection drug use and homelessness. Modern echocardiographic techniques have considerably augmented our ability to diagnose IE and to understand its natural history. Despite this, there are many areas in which our understanding of right sided IE remains incomplete. ${ }^{3}$
\end{abstract}

(Cardiovasc.j. 2010; 3(1) : 101-103)

\section{Case History:}

Mr I, 22 years aged businessman from kishorgonj presented with history of fever for 21 days, shortness of breath for 5 days. Fever was associated with polyarthritis, sweating and weight loss. His shortness of breath was progressive in nature and it was associated with haemoptysis. Patient also developed dependent edema in last one week. He had no history suggestive of rheumatic fever or congenital heart disease. Mr I was an intravenous drug abuser. His primary care physician has done some routine investigations which revealed neutrophilic leucocytosis and military-mottling shadows in chest X-ray. And he was diagnosed as case of disseminated tuberculosis. But he was not responding to anti - TB drugs. Later on he was examined by a cardiologist and noticed a murmur in the precordium and he was referred to National Institute of Cardiovascular Diseases.

On physical examination, patient was ill-looking, febrile and edematous. There was a murmur suggestive of tricuspid regurgitation. Patient also had hepatomegaly and coarse crepitation over the both lung fields.

Investigations revealed neutrophilic leucocytosis with haematuria and albuminuria. Echocardiography revealed RA and RV are dilated with a large mass of grape like vegetations attached to tricuspid valve leaflets. On blood culture, there was growth of coagulase negative Staphylococcus aureus. Mr I was treated with I/V antibiotics for four weeks. At the end of treatment he was afebrile with good general wellbeing and had moderate tricuspid regurgitation

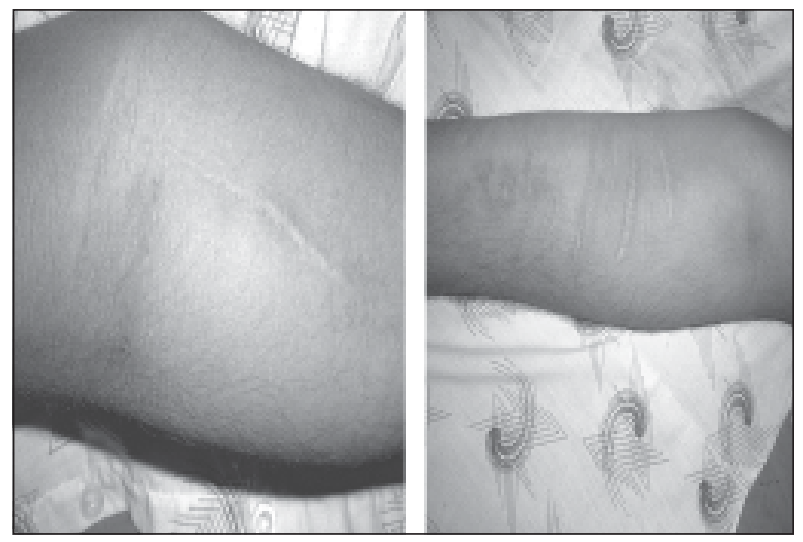

Fig.-1: Scar marks in the forearm at the injection sites.

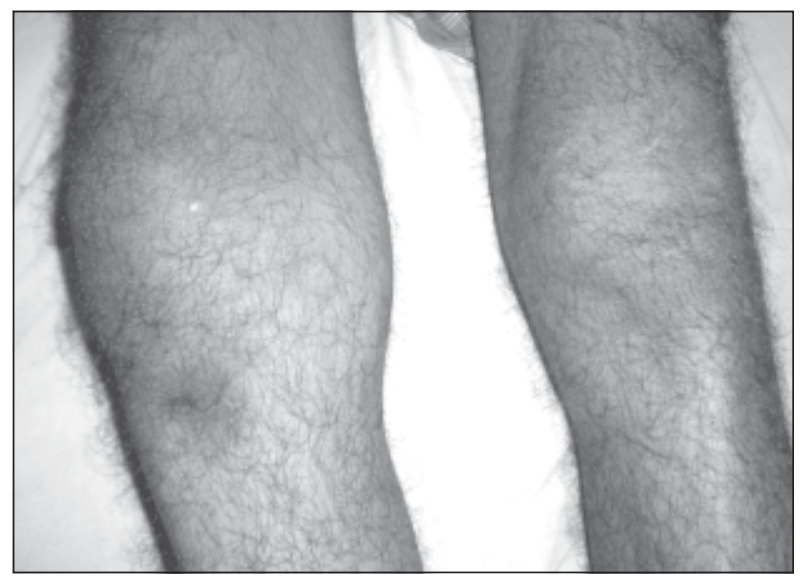

Fig-2: Swollen right knee joint. 


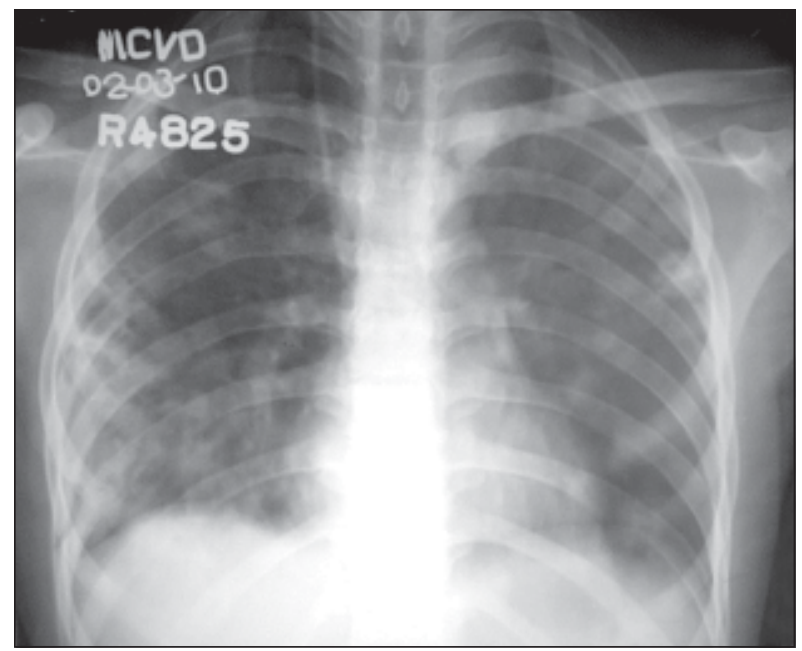

Fig.-3: Chest X-ray showing bilateral pulmonary infiltrates.

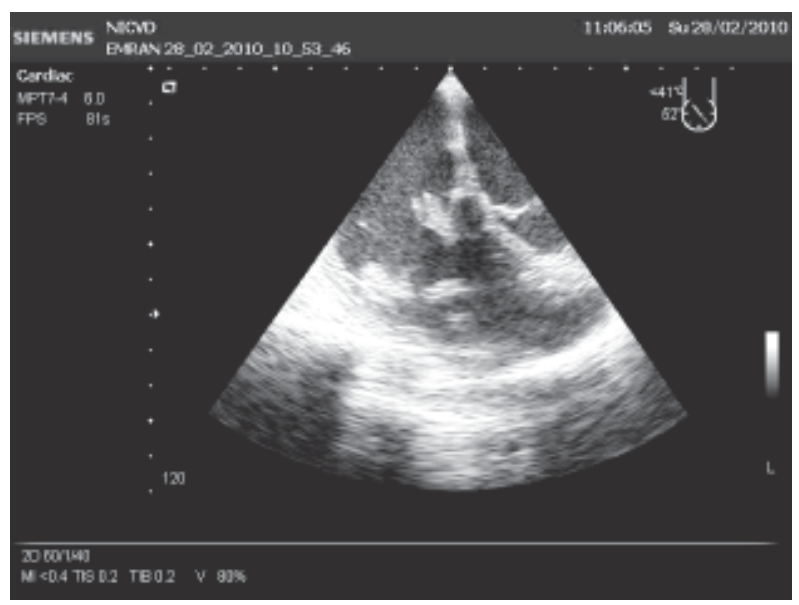

Fig.-4: Echocardiography showing vegetation in Tricuspid valve.

\section{Discussion :}

Among injection drug users (IVDU) presenting with fever, 13\% will have Echocardiographic evidence of IE. ${ }^{2}$ If injection drug users with bacteraemia from an inner city demographic are considered, up to $41 \%$ will have evidence of IE. ${ }^{3}$ In keeping with the demographic characteristics of injection drug use, this disease occurs most commonly in young males in their 20 s and 30 s. In our case, patient was an intravenous drug abuser for last three years. So, infective endocarditis should have been one of the probable diagnoses on the beginning. But due to lack of adequate history regarding his drug abuse habit and lack of suspicion for infective endocarditis, the diagnosis was delayed which could be a fatal mistake.
Tricuspid valve was the only valve involved in our patient. In most of the cases of infective endocarditis in IVDU tricuspid valve is involed. In one study of such cases resulted, $86 \%$ patients had exclusively right sided, while $14 \%$ had left sided involvement. ${ }^{3}$ In some recent reports, however, the incidence of left and right sided endocarditis in this population has been similar. ${ }^{4}$ The tricuspid valve is most frequently involved followed by the mitral and then the aortic valve. Concurrent involvement of both left and right sided valves is not infrequent. ${ }^{4}$ In right sided IE it is almost always the tricuspid valve that is involved. One study of injection drug users found vegetations involving the tricuspid valve in 127 episodes, the pulmonary valve in four, and both valves in one instance. ${ }^{5}$

The causative organism in our patient was Satph. Aureus. The dominant infective organism is Staphylococcus aureus, in most series accounting for around $70 \%$ of infections, with the remainder being caused mainly by streptococcal species or, less frequently, by Gram negative organisms, fungi or diphtheroids. Pseudomonal infections may have a predilection for multi-valve involvement. Infections with unusual organisms and polymicrobial infections do occur, probably as the result of the injection of contaminated diluent, but are relatively unusual. Blood cultures are positive in a high proportion of cases of right sided IE. The reported prevalence of HIV disease in patients with right sided IE is variable. In a Spanish series of injection drug users with IE, $76 \%$ were HIV positive. ${ }^{6}$ But our patient was HIV negative.

Common complication of right sided infective endocarditis is pulmonary embolism, which was present in our patient. Septic pulmonary embolism was responsible for haemoptysis. The features in the chest $X$ - ray were misinterpreted as Tuberculosis. In fact chest $\mathrm{x}$ ray is a helpful laboratory aid in diagnosing right sided endocarditis, with $55 \%$ of radiographs at presentation showing evidence of pulmonary infiltrates compatible with pulmonary emboli. ${ }^{5}$

Transthoracic and transesophageal echocardiography showed large vegetations in RA nad floating into the RV. Tricuspid vegetations are often large and may be in excess of $2 \mathrm{~cm}$, a finding that does not necessarily suggest the presence of fungal endocarditis. On rare occasions, vegetations may 
be sufficiently large so as to be confused with an intracardiac tumour. As with vegetations on the left sided valves, they tend to be localised to the atrial side of the valve in the path of the regurgitant jet. $^{3}$

The patient was successfully treated with intravenous Vancomycin and Gentamicin for four weeks. These patients may be difficult and disruptive and often engender negative attitudes in health care professionals. Their lack of adherence to in-hospital treatment is a major practical hurdle. These patients often develop withdrawal symptoms. Methadone and/or other agents may be needed to control withdrawal symptoms. For these reason shorter courses of treatment schedule has been developed. Intravenous Cloxacillin $2 \mathrm{~g}$ every four hours for two weeks together with Gentamicin $1 \mathrm{mg} / \mathrm{kg}$ every eight hours is effective for tricuspid endocarditis caused by methicillin sensitive staphylococcal species. In the presence of a relative or absolute contraindication to gentamicin, therefore, a two week course of Cloxacillin alone may be adequate. In the event that the patient cannot be persuaded to stay in hospital for the full two weeks, a 28 day course of oral treatment with Ciprofloxacin and Rifampin is prescribed, ideally with administration in a supervised setting such as a drug treatment facility. ${ }^{7}$

Most patients can be treated medically, with surgery being necessary in only a small minority of cases. ${ }^{5}$ Indications for surgical treatment are better defined for left than for right sided endocarditis. Sepsis, which is uncontrolled despite adequate antibiotic treatment, and intractable right heart failure despite appropriate medical treatment, are the most important indications for surgical intervention. The finding of a paravalvar abscess or fungal endocarditis is usually indications for surgery. Recurrent pulmonary embolism following the initiation of antibiotic treatment may occur and does not in itself represent an indication for surgery. ${ }^{5}$ Some authors have felt that large vegetation size (diameter $>10$ $\mathrm{mm}$ ) in the context of persisting fever identifies a set of patients who are likely to benefit from surgery. ${ }^{8}$ Others have felt that many of these patients will eventually recover with medical treatment. ${ }^{9}$ Very large vegetations (> $\left.20 \mathrm{~mm}\right)$, however, are associated with a poor outcome. ${ }^{5}$ Patients may also require an operation to treat concomitant left sided endocarditis.

\section{Conclusion :}

In patients with fever and a history of intravenous drug abuse, the possibility of infective endocarditis should be considered; especially if it is associated with a right sided murmur. Most of the patients can be successfully treated with I/V or even oral antibiotics, with only $10 \%$ mortality. It can be prevented by reducing intravenous drug abuse. And by harm reduction strategies aimed at reducing injection frequency (such as methadone availability) or clean injection strategies (clean injection education, injection rooms, needle exchange programmes or legal heroin).

\section{References:}

1. Hoen B, Alla F, Selton-Suty C, et al. Changing profile of infective endocarditis: results of a 1-year survey in France. JAMA 2002;288:75-81.

2. Weisse AB, Heller DR, Schimenti RJ, et al. The febrile parenteral drug user: a prospective study in 121 patients. Am J Med 1993;94:274-80.

3. Moss R,Munt B. Injection drug use and right sided endocarditis. Heart 2003; 89: 577-581.

4. Mathew J, Addai T, Anand A, et al. Clinical features, site of involvement, bacteriologic findings, and outcome of infective endocarditis in intravenous drug users. Arch Intern Med 1995;155:1641-8.

5. Hecht S, Berger M. Right-sided endocarditis in intravenous drug users. Prognostic features in 102 episodes. Ann Intern Med 1992;117:560-6.

6. Ribera E, Miro JM, Cortes E, et al. Influence of human immunodeficiency virus 1 infection and degree of immunosuppression in the clinical characteristics and outcome of infective endocarditis in intravenous drug users. Arch Intern Med 1998;158:2043-50.

7. Heldman AW, Hartert TV, Ray SC, et al. Oral antibiotic treatment of right-sided staphylococcal endocarditis in injection drug users: prospective randomized comparison with parenteral therapy. Am J Med 1996;101:68-76.

8. Robbins M, Frater R, Soeiro R, et al. Influence of vegetation size on clinical outcome of right-sided infective endocarditis. Am J Med 1986;80:165-71.

9. Manolis AS, Melita H. Echocardiographic and clinical correlates in drug addicts with infective endocarditis. Implications of vegetation size. Arch Intern Med 1988;148:2461-5. 